
\title{
28 Research Suare \\ Effect of three therapeutic strategies for FIGO stage IB2/IIA2 cervical Cancer
}

\section{yong zhang}

First Affiliated Hospital of Guangxi Medical University

\section{Xiaobi Tang}

liuzhou people's hospital

\section{Meizhu Shen}

First Affiliated Hospital of Guangxi Medical University

\section{Shanshan Ma}

First Affiliated Hospital of Guangxi Medical University

\section{Wenchao Yuan}

First Affiliated Hospital of Guangxi Medical University

Rensheng Wang ( $\sim 13807806008 @ 163 . c o m$ )

Fang Wu

First Affiliated Hospital of Guangxi Medical University

\section{Research}

Keywords: cervical cancer, neoadjuvant chemotherapy, concurrent chemoradiotherapy, toxicity, survival

Posted Date: April 15th, 2020

DOI: https://doi.org/10.21203/rs.3.rs-22534/v1

License: (c) (i) This work is licensed under a Creative Commons Attribution 4.0 International License.

Read Full License 


\section{Abstract}

Background: To evaluate the long-term efficacy and toxicities of three therapeutic strategies for patients with International Federation of Gynecology and Obstetrics (FIGO) stage IB2/IIA2 cervical cancer.

Methods : 206 patients with stage IB2/IIA2 cervical cancer were selected in this retrospective study. The patients were classed into three groups according to the primary therapy: primary surgical treatment (PST), neoadjuvant chemotherapy followed by radical surgery (NAC+RS), and concurrent chemoradiotherapy (CCRT). We observed the inter-group differences in patient characteristics, surgery approaches, postoperative rick factors, supplemental therapies and overall survival (OS), disease-free survival (DFS) and acute and late toxicities.

Results: Less patients in NAC+RS group had deep cervical stromal invasion than those in PST group $(p=0.024)$. No differences in lymph node metastasis, intravascular tumor embolus, vaginal margin and the need of postoperative supplement treatment were observed between PST and NAC+RS ( $P=0.734, P$ $=0.704, P=0.487$ and $P=0.714$, respectively). With a median follow-up time of 57 months, the 3-year DFS and OS in PST, NAC+RS and CCRT were $85.6 \%, 79.2 \%, 85.7 \%$ and $87.5 \%, 84.9 \%, 85.7 \%$ ( $P=0.424$ and $P$ $=0.856$, respectively). The most frequently observed acute toxicities were hematologic side effects. No significant inter-group differences in leukopenia, neutropenia, thrombocytopenia and anemia were observed (all $\mathrm{P}>0.05$ ). No patient experienced grade $3-4$ hepatotoxicity and nephrotoxic. Late toxicity $\geq$ grade 3 mainly consisted of lower extremity lymphedema (4/104, 3.8\%), bowel obstruction (3/104, $2.9 \%)$ and thrombosis $(1 / 104,1.0 \%)$ in PST, lower extremity lymphedema $(5 / 53,9.4 \%)$ and bowel obstruction $(1 / 53,1.9 \%)$ in NAC+RS and radiation proctitis $(4 / 49,8.2 \%)$ and femoral head necrosis (1/49, $2.0 \%)$ in CCRT. No grade 5 toxicities were observed. There was no significant difference in cumulative late adverse effects rate in the three groups $(P=0.777)$. The patients in NAC+RS had increased hospitalization cost than that in PST $(P=0.000)$ and CCRT $(P=0.000)$ and prolonged hospitalization time than that in PST $(P=0.000)$ and same as that in CCRT $(P=0.07)$.

Conclusion: Preoperative NAC decreased the incidence of deep cervical stromal invasion but had no effect on other high risk factors and the need of postoperative supplement treatment. No inter-group differences were demonstrated in 3-year DFS and OS and cumulative acute and late adverse effects. NAC+RS would need longer hospitalization time and cost more. Nevertheless, we believe it should be further explored in prospective trials.

\section{Background}

Cervical cancer is one of the common malignant tumors that affect the health of females all over the world [1]. Concurrent chemoradiotherapy has been the standard treatment of locally advanced cervical cancer (LACC) since 1999 [2-6]. However, $25 \%$ to $40 \%$ of patients still experience relapse, and some experience distant failure despite local control after chemoradiation [7]. Patients with tumors measuring $>4 \mathrm{~cm}$ in the largest diameter have a worse prognosis compared to those with smaller tumors, regardless 
of treatment $[8,9]$. Additionally, the optimal therapeutic strategies for patients with stage IB2/IIA2 cervical cancer remains controversial. Since the 1980s, neoadjuvant chemotherapy (NAC) followed by radical surgery (RS) or Concurrent chemoradiotherapy (CCRT) has been proposed and carried out for LACC [1012]. The rationale for NAC is that this approach would reduce tumor volume, kill subclinical lesions, increase tumor resectability, eliminate micro-metastases, and has received widespread attention. In addition, chemotherapy given in the neoadjuvant setting might be more effective, partly because it is delivered to uncompromised tumor blood supply and to a population of chemosensitive tumor cells. Therefore, it has emerged as an alternative option of treatment based on several studies that have shown a significant benefit of NAC+RS over radiotherapy alone or concurrent chemotherapy in terms of overall survival (OS) and disease free survival (DFS) $[13,14]$. On the other hand, some studies have reported inconsistent findings regarding the outcomes of NAC $[15,16]$.

Therefore, the aim of this study was to evaluate the long-term efficacy and toxicities of three therapeutic strategies including primary surgical treatment (PST), neoadjuvant chemotherapy followed by radical surgery (NAC+RS), and concurrent chemoradiotherapy (CCRT) for patients with 2009 International Federation of Gynecology and Obstetrics (FIGO) stage IB2/IIA2 cervical cancer, and provide a theoretical basis for the clinical treatment.

\section{Methods}

\section{Patients}

From April 2010 to December 2015, 206 patients with 2009 FIGO stage IB2, IIA2 cervical cancer treated in our hospital were included in the study. Eligibility criteria included newly diagnosed patients between 18 and 65 years of age with histopathologically confirmed carcinoma of the uterine cervix; Eastern Cooperative Oncology Group (ECOG) performance status of 1 or less; normal haematologic values; good liver and renal function; no evidence of secondary cancer. Computed tomography (CT) or magnetic resonance imaging (MRI) was performed before therapy to assess tumor extension and exclude distant metastasis.

This study was approved by the Medical Ethics Committee of First Affiliated Hospital of Guangxi Medical University. All patients provided informed consent.

\section{Neoadjuvant Chemotherapy (nac)}

Patients in NAC + RS underwent NAC as follows: 1 . cisplatin or nedaplatin $60 \mathrm{mg} / \mathrm{m} 2$ on day 1 with paclitaxel $175 \mathrm{mg} / \mathrm{m} 2$ on day 1 every 3 weeks for 1 to 3 cycles; 2 . cisplatin or nedaplatin $60 \mathrm{mg} / \mathrm{m} 2$ on day 1 with docetaxel $75 \mathrm{mg} / \mathrm{m} 2$ on day 1 every 3 weeks for 1 to 3 cycles; 3 . cisplatin or nedaplatin $60 \mathrm{mg} / \mathrm{m} 2$ on day 1 with irinotecan $60 \mathrm{mg} / \mathrm{m} 2$ on day 1,8 and 15 every 3 weeks for 1 to 3 cycles. 


\section{Surgery}

Type III radical hysterectomy plus bilateral pelvic lymphadenectomy was performed within 1 to 2 weeks of diagnosis in PST group and within 2 to 4 weeks after the last administration of chemotherapy in NAC group. Postoperative supplemental chemoradiotherapy was administered based on the histopathologic results in accordance with the National Comprehensive Cancer Network guideline.

\section{Concurrent chemoradiotherapy (CCRT)}

External beam radiotherapy (EBRT) to a dose of $45 \mathrm{~Gy} \sim 50 \mathrm{~Gy}$ in 25 fractions was delivered using IMRT technique. Brachytherapy was given within 1 week of completion of EBRT by Microselectron HDR unit (Elekta, Veenendaal, Netherlands). A dose of $28 \mathrm{~Gy}$ in 4 fractions was delivered to point A at weekly intervals. Cisplatin or nedaplatin at a dose of $40 \mathrm{mg} / \mathrm{m} 2$ was administered concurrently once a week during EBRT.

As anti-emetic agents, the combination of a steroid and ondansetron hydrochloride or granisetron hydrochloride was administered before chemotherapy. Prophylactic use of recombinant granulocyte colony-stimulating factor was not allowed.

\section{Evaluation during treatment and Follow-up}

All patients were evaluated once a week during treatment. At the completion of treatment, patients were evaluated clinically and radiologically using a contrast-enhanced CT scan or MRI of abdomen and pelvis. Then patients were follow-up every 3 months during the first 2 years, every 6 months from 3 to 5 years, and every 1 year thereafter. Acute and late treatment-related morbidities were assessed according to the Common Terminology Criteria for Adverse Events (CTCAE) version 5.0. The surgical approach, hospitalization time and treatment cost were also recorded.

\section{Statistical analysis}

Statistical comparisons of inter-group differences in characteristics and the post-operatic adjuvant therapy rate, as well as the incidence of acute and late toxicity, were performed using the Chi-square test or Fisher exact test. Hospitalization time and treatment cost in three groups were compared by variance analysis. Survival was analyzed using the Kaplan-Meier method and log-rank test. All data were analyzed using the SPSS Statistics 22.0. $P<0.05$ was considered statistically significant.

\section{Results}

\section{Patients And Baseline Characteristics}


Of the 206 patients, 126 had 2009 stage IB2, 80 had stage IIA2. The mean age of the patients was 46 years (range 22-69 years). Pathological type distributions were as follows: squamous carcinoma, 174; non-squamous carcinoma, 32. Of all the patients, 104, 53 and 49 cases were classified into the PST, NAC + RS and CCRT groups, respectively. No significant difference was detected in surgical approach between the PST and NAC + RS groups. Table 1 list the clinical characteristics of the patients.

Table 1

Patient characteristics

\begin{tabular}{|c|c|c|c|c|}
\hline Characteristics & $\begin{array}{l}\text { PST } \\
(n=104)\end{array}$ & $\begin{array}{l}\text { NACT } \\
(n=53)\end{array}$ & $\begin{array}{l}\text { CCRT } \\
(n=49)\end{array}$ & $P$ \\
\hline Age(years) & & & & 0.090 \\
\hline$>45$ & 54 & 27 & 34 & \\
\hline$\leq 45$ & 50 & 26 & 15 & \\
\hline Hemoglobin & & & & 0.077 \\
\hline$\geq 115 \mathrm{~g} / \mathrm{L}$ & 52 & 25 & 33 & \\
\hline$<115 \mathrm{~g} / \mathrm{L}$ & 52 & 28 & 16 & \\
\hline FIGO Stage & & & & 0.054 \\
\hline IB2 & 70 & 33 & 23 & \\
\hline$\| A 2$ & 34 & 20 & 26 & \\
\hline Differentiation degree & & & & 0.357 \\
\hline Grade1 & 4 & 1 & 0 & \\
\hline Grade2 & 72 & 40 & 39 & \\
\hline Grade3 & 28 & 12 & 10 & \\
\hline Histology Type & & & & 0.072 \\
\hline SCC & 82 & 47 & 45 & \\
\hline Non-SCC & 22 & 6 & 4 & \\
\hline Surgical Approach & & & & 0.082 \\
\hline Laparotomy & 82 & 35 & - & \\
\hline
\end{tabular}




\section{High-risk factors (HRFs) predictive of supplement treatment}

Less patients in NAC + RS group had deep cervical stromal invasion than those in the PST $(P=0.024)$. No differences in lymph node metastasis, intravascular tumor embolus and vaginal margin were observed between PST and NAC + RS group ( $P=0.734, P=0.704$ and $P=0.487$, respectively). There was no difference in the rate of postoperative supplement treatment $(P=0.714)$ (Table 2).

Table 2

Postoperative HRFs associated with pathology

\begin{tabular}{|c|c|c|c|}
\hline Characteristics & $\begin{array}{l}\text { PST } \\
n(\%)\end{array}$ & $\begin{array}{l}\text { NACT + RS } \\
\text { n (\%) }\end{array}$ & $P$ \\
\hline Lymph node metastasis & & & 0.734 \\
\hline Negative & $81(77.9)$ & $40(75.5)$ & \\
\hline Positive & $23(22.1)$ & $13(24.5)$ & \\
\hline Intravascular tumor embolus & & & 0.704 \\
\hline No & $90(86.5)$ & $47(88.7)$ & \\
\hline Yes & $14(13.5)$ & $6(11.3)$ & \\
\hline Vaginal margin & & & 0.487 \\
\hline Negative & $102(98.1)$ & $51(96.2)$ & \\
\hline Positive & $2(1.9)$ & $2(3.8)$ & \\
\hline Deep stromal invasion & & & 0.024 \\
\hline No & $25(24.0)$ & $22(41.5)$ & \\
\hline Yes & $79(76.0)$ & $31(58.5)$ & \\
\hline Supplemental treatment & & & 0.714 \\
\hline Non-treatment & $30(28.8)$ & $11(20.8)$ & \\
\hline Radiotherapy & $27(26.0)$ & $15(28.3)$ & \\
\hline Chemoradiotherap & $43(41.3)$ & $24(45.3)$ & \\
\hline Chemotherapy & $4(3.9)$ & $3(5.6)$ & \\
\hline
\end{tabular}




\section{Survival Analysis}

The median follow-up time was 57 months (range 4-104 months). 28 patients died: 13 in PST group, 8 in NAC + RS and 7 in CCRT. The median time of death of the 28 patients was 14 months (range 633 months). For all patients, the 3-year OS was $86.4 \%$. There was no significant difference in the three groups (PST: $87.5 \%$, NAC + RS: $84.9 \%$, CCRT: $85.7 \%, P=0.843$ ) (Fig. 1).

\section{Patterns Of Treatment Failures}

There were 32 patients who experienced treatment failures. Local recurrence was observed in 13 patients (4 in PST, 7 in NAC + RS and 2 in CCRT, respectively) after a median time of 14 months (range 1033 months) in PST, 9.5 months (range 6-29 months) in NAC + RS, 10 months (range 8-15 months) in CCRT, respectively. 12 patients experienced distant metastasis (6 in PST, 3 in NAC + RS and 3 in CCRT, respectively) after a median time of 14 months (range 10-27 months) in PST, 9 months (range 629 months) in NAC + RS, 14 months (range 7-21 months) in CCRT, respectively. Local recurrence and distant metastasis were presented in 7 patients (4 in PST, 1 in NAC + RS and 2 in CCRT, respectively) after a median time of 12 months (range 10-14 months), 29 months and 11 months (range 8-15 months), respectively. For all patients, the 3-year DFS rates were $84.0 \%$. There was no significant difference in the three groups (PST: $85.6 \%$, NAC + RS: 79.2\%, CCRT: 85.7\%, $P=0.414$ ) (Fig. 2).

\section{Acute And Late Toxicities}

The most frequently observed acute toxicities were hematologic side effects. 11 patients $(11.5 \%)$ in PST, $12(27.9 \%)$ in NAC + RS and $17(39.5 \%)$ in CCRT experienced grade $3-4$ leukopenia $(P=0.248) .10$ patients (10.4\%) in PST, 11 (25.6\%) in NAC + RS, 12 (27.9\%) in CCRT underwent grade 3-4 neutropenia ( $P$ $=0.435) .3(3.1 \%)$ in PST, $1(2.3 \%)$ in NAC + RS, 1 (2.3\%) in CCRT suffered grade $3-4$ thrombocytopenia $(P$ $=0.116) .4(4.2 \%)$ in PST, 11 (25.6\%) in NAC + RS, 6 (14.0\%) in CCRT had grade $3-4$ anemia $(P=0.190)$. No grade 3-4 hepatotoxicity and nephrotoxic were observed. Detail data was shown in Table 3. Grade 34 late toxicity mainly consisted of lower extremity lymphedema (4/104, 3.8\%), bowel obstruction (3/104, $2.9 \%)$ and thrombosis $(1 / 104,1.0 \%)$ in PST, lower extremity lymphedema $(5 / 53,9.4 \%)$ and bowel obstruction $(1 / 53,1.9 \%)$ in NAC + RS and radiation-induced rectitis $(4 / 49,8.2 \%)$ and femoral head necrosis $(1 / 49,2.0 \%)$ in CCRT. No grade 5 toxicities were observed. There was no significant difference in cumulative late adverse effects rate in the three groups $(P=0.777)$. 
Table 3

Frequency of acute toxicities

\begin{tabular}{|c|c|c|c|c|c|c|c|}
\hline \multirow[t]{2}{*}{ Toxicity } & \multicolumn{2}{|l|}{$\begin{array}{l}\text { PST } \\
\text { n (\%) }\end{array}$} & \multicolumn{2}{|c|}{$\begin{array}{l}\text { NACT + RS } \\
n(\%)\end{array}$} & \multicolumn{2}{|l|}{$\begin{array}{l}\text { CCRT } \\
\text { n (\%) }\end{array}$} & $P$ \\
\hline & G1-2 & G3-4 & G1-2 & G3-4 & G1-2 & G3-4 & - \\
\hline Leukopenia & $\begin{array}{l}30 \\
(31.3)\end{array}$ & $\begin{array}{l}11 \\
(11.5)\end{array}$ & $\begin{array}{l}17 \\
(39.5)\end{array}$ & $\begin{array}{l}12 \\
(27.9)\end{array}$ & $\begin{array}{l}22 \\
(51.2)\end{array}$ & $\begin{array}{l}17 \\
(39.5)\end{array}$ & 0.248 \\
\hline Neutropenia & $\begin{array}{l}21 \\
(21.9)\end{array}$ & $\begin{array}{l}10 \\
(10.4)\end{array}$ & $\begin{array}{l}12 \\
(27.9)\end{array}$ & $\begin{array}{l}11 \\
(25.6)\end{array}$ & $\begin{array}{l}24 \\
(55.8)\end{array}$ & $\begin{array}{l}12 \\
(27.9)\end{array}$ & 0.435 \\
\hline Thrombocytopenia & $6(6.3)$ & $3(3.1)$ & $3(7.0)$ & $1(2.3)$ & $\begin{array}{l}17 \\
(39.5)\end{array}$ & $1(2.3)$ & 0.116 \\
\hline Anemia & $9(9.4)$ & $4(4.2)$ & $7(16.3)$ & $\begin{array}{l}11 \\
(25.6)\end{array}$ & $\begin{array}{l}10 \\
(23.3)\end{array}$ & $6(14.0)$ & 0.190 \\
\hline Hepatotoxicity & $5(5.2)$ & 0 & $4(9.3)$ & 0 & $5(11.6)$ & 0 & 0.931 \\
\hline
\end{tabular}

\section{Hospitalization Time And Cost}

The average hospitalization time was 49.72 days in PST, 63.08 in NAC and 54.22 in CCRT, respectively ( $P=0.000)$. The patients in NAC + RS needed longer hospitalization time than those in PST $(P=0.000)$ but had same length of stay as those in CCRT $(P=0.07)$. The average hospitalization expenditure was $\$ 7969.77$ in PST, $\$ 9915.97$ in NAC + RS, and \$7646.62 in CCRT, respectively $(P=0.000)$. NAC + RS had increased hospitalization cost compared with PST $(P=0.000)$ and CCRT $(P=0.000)$. Detail data was shown in Table 4.

Table 4

Length of hospital stay and hospitalization expenditure

\begin{tabular}{|lllll|}
\hline & $\begin{array}{l}\text { PST } \\
(\mathbf{n = 1 0 4 )}\end{array}$ & $\begin{array}{l}\text { NACT + RS } \\
(\mathbf{n = 5 3 )}\end{array}$ & $\begin{array}{l}\text { CCRT } \\
(\mathbf{n = 4 9 )}\end{array}$ & $P$ \\
\hline Hospitalization time(days) & $49.72 \pm 19.40$ & $63.08 \pm 15.26$ & $54.22 \pm 8.50$ & 0.000 \\
\hline $\begin{array}{l}\text { Hospitalization } \\
\text { expenditure(\$) }\end{array}$ & $\begin{array}{l}7969.77 \pm \\
3115.07\end{array}$ & $\begin{array}{l}9915.97 \pm \\
2553.34\end{array}$ & $\begin{array}{l}7646.62 \pm \\
2872.94\end{array}$ & 0.000 \\
\hline $\begin{array}{l}P S T \text { Primary surgical treatment, } \\
\text { CCRT Concurrent chemoradiotherapy }\end{array}$ & & & \\
\hline
\end{tabular}




\section{Discussion}

Although platin based chemoradiation (CRT) has been the standard treatment for patients with locally advanced cervical cancer since 1999 [2-6]. Patients with tumors measuring $>4 \mathrm{~cm}$ in the greatest diameter have a worse prognosis and the optimal management for patients with stage IB2/IIA2 cervical cancer remains controversial and ambiguous. Chen et al. reported that 142 patients with locally advanced cervical cancer (stage IB2-IIB, tumor diameter $>$ or $=4 \mathrm{~cm}$ ) were randomly assigned to receive either preoperative neoadjuvant chemotherapy (NAC) followed by surgery or primary surgery directly [17]. Significantly reduced pelvic lymph node metastasis and parametrial infiltration rates were detected in the NAC group compared with the primary surgery group $(25.0 \%$ vs. $42.9 \%, P=0.025 ; 25.0 \%$ vs. $41.4 \%, P=$ 0.038 , respectively). A randomized multicenter study from Yang et al. that sought to evaluate the toxicity and curative effect of NAC for stage Ib2, Ila2, and Ilb cervical cancer patients found that deep stromal invasion and lymphovascular space invasion (LVSI) were significantly less in the NAC group compared to the DS ( directly with surgery) group $(P=0.002)$, but there was no difference in lymph node metastasis $(P$ $=0.698)$ or positive parametrial involvement $(P=0.469)$ [18]. The rate of postoperative radiotherapy in the NAC group was lower than that of the DS group, although the difference was not significant $(58.9 \%$ vs. $63.3 \%, P=0.472$ ). In the present study, the rate of deep stromal invasion in NAC group was significantly lower than that in the PST group, while no significant difference was detected in lymph node metastasis between the two groups. This finding is consistent with the literature [18] and indicates that NAC is effective in reducing risk factors of recurrence. A meta-analysis by Kim et al. showed that the use of NAC in FIGO stage IB1-IIA cervical cancer decreased the incidence of risk factors such as large tumor size ( $\geq$ $4 \mathrm{~cm}$ ) and lymph node metastasis when compared to radical surgery in all studies and randomized controlled trials (RCTs) [19]. As a result, NAC reduced the need for adjuvant radiotherapy. In our study, although the NAC group was superior to the PST group in terms of deep stromal invasion, the groups did not differ significantly with respect to other high-risk factors. Additionally, we observed no inter-group differences in the rates of supplemental radiotherapy and chemotherapy. We might thus consider that the standards used to determine the need for adjuvant treatment may not have been uniform. Additionally, a local tumor with a largest diameter $>4 \mathrm{~cm}$ is itself a risk factor for recurrence. Therefore, the gynecologic oncologist may still opt for supplemental therapy even in the absence of postoperative high risk factors. Besides, we observed no difference in surgical route between the NAC and PST groups. However, a multicenter retrospective clinical study of FIGO stage IB2 or IIA2 cervical cancer by Zhao et al. demonstrated that the NAC and PST groups differed in terms of the surgical route, with the former favoring laparoscopic procedures [16]. The inconsistence may be attributable to the small sample study from single-institution by us.

In previous reports, the effect of NAC on improving survival remains controversial. A meta-analysis had showed that neoadjuvant chemotherapy (NAC) plus radical surgery significantly improved OS, and decreased local and distant recurrence rates compared to radical surgery alone in selected patients with locally advanced cervical cancer (FIGO stage IB2-IIB) [13]. However, the relationship between NAC and longer DFS could not be demonstrated and further research was urgently needed to confirm it. Gupta et al.'s research showed that the 5-year DFS in patients with stage IB2, IIA, or IIB cervical cancer in the 
neoadjuvant chemotherapy plus surgery group was $69.3 \%$ compared with $76.7 \%$ in the concomitant chemoradiation group $(P=0.038)$, whereas the corresponding 5 -year OS rates were $75.4 \%$ and $74.7 \%$, respectively $(P=0.87)$ [14]. A retrospective cohort study from Yan et al. grouped the patients with cervical carcinoma stage IB2 or IIA2 according to whether they received NAC or not before surgery and evaluated the treatment and prognosis of NAC [15]. The results showed that NAC did not significantly affect DFS ( $P$ $=0.453)$ and OS ( $P=0.933)$ between the 2 groups. In a multi-center retrospective clinical study, Zhao et al. reported no significant difference in 5-year cumulative survival rate between the NAC and PST groups (83.3\% versus $87.2 \%, P=0.418$ ) [16]. In this study, we observed no inter-group differences in 3-year DFS and OS, with the DFS of $85.6 \%, 79.2 \%$ and $85.7 \%$ in PST, NAC and CCRT, respectively $(P=0.414)$ and OS of $87.5 \%, 84.9 \%$ and $85.7 \%(P=0.843)$. Thus it can be seen that some studies have supported the use of NAC, whereas others found no evidence of an effect of NAC for locally advanced cervical cancer.

A meta-analysis by Marchetti et al. shown severe acute toxicity in the CCRT group compared with neoadjuvant chemotherapy followed by surgery (NAC + S) in stage IB2, IIA and IIB cervical cancer (1994 FIGO stage) [20]. In the present study, the main acute adverse effect was hematology toxicity but no significant differences in the three groups were observed. The inconsistence to other studies may be attributed to different chemotherapy regimens. Accumulative incidence of grade 3-4 late adverse effects in the three groups were not high and the inter-group difference was not significant. Impact on long term toxicity and quality of life remained to be proven.

Several retrospective analyses suggest that prolonged RT treatment duration has an adverse effect on outcome [21-25]. Extending the overall treatment beyond 6 to 8 weeks can result in approximately a 0.5$1 \%$ decrease in pelvic control and cause specific survival for each extra day of overall treatment time. Thus, it is generally accepted that the entire RT course (including both EBRT and brachytherapy components) should be completed in a timely fashion (within 8 weeks). In the present study, the average overall treatment time was less than 8 weeks and met the requirement of designed proposal. Although there was no study to explore the impact of treatment time of NAC followed by RS on tumor control, we found that NAC + RS prolonged the treatment time and as a result increased the hospitalization cost than that in patients treated with CCRT. The impact of treatment time and expenditure on clinical outcome remained to be proven.

This study has some limitations. One limitation is that it is a small sample single-center retrospective study. The follow-up period is not enough. Because the original text did not provide enough data, no analysis was done about response rate, which is the most important prerequisite for improvement of curative effects. Quality of life was not prospectively measured. Randomize trials need to be conducted to explore the effect on clinical outcome in patients with different treatments.

\section{Conclusion}

Preoperative NAC decreased the incidence of deep cervical stromal invasion but had no effect on other high risk factors and the need of postoperative supplement treatment. No inter-group differences were 
demonstrated in 3-year DFS, OS and cumulative acute and late adverse effects. NAC + RS would need longer hospitalization time and cost more. Randomize trials need to be conducted to explore the effect on clinical outcome in patients with different treatments.

\section{Abbreviations}

FIGO: International Federation of Gynecology and Obstetrics; PST: Primary surgical treatment; NACT: Neoadjuvant chemotherapy; RS: Radical surgery; CCRT: Concurrent chemoradiotherapy; OS: Overall survival; DFS: Disease-free survival; LACC: Locally advanced cervical cancer; ECOG: Eastern Cooperative Oncology Group; CT: Computed tomography; MRI: Magnetic resonance imaging; EBRT: External beam radiotherapy; CTCAE: Common Terminology Criteria for Adverse Events; RCTs: Randomized controlled trials

\section{Declarations}

\section{Acknowledgments}

Not applicable.

\section{Authors' contributions}

$Y Z, R S W$ and FW designed the study and reviewed and/or revised of the manuscript. YZ, XBT, SMZ and FW managed patients, analyzed clinic outcome, performed the statistical analysis and wrote the manuscript. XBT, SMZ, SSM and WCY conducted the follow-up. All authors read and approved the final manuscript.

\section{Funding}

This work was supported by the Program for Improvement of Scientific Research Ability of Young and Middle-Aged Scholars of Higher Education of Guangxi, China (2017KY0104) and Project of Development and Application of Appropriate Medical and Health Technologies in Guangxi, China (S2018025).

\section{Availability of data and materials}

All data generated or analysed during this study are included in this published article.

\section{Ethics approval and consent to participate}

This study was approved by the Medical Ethics Committee of First Affiliated Hospital of Guangxi Medical University. All patients provided informed consent.

\section{Consent for publication}

Not applicable. 


\section{Competing interests}

The authors declare that they have no competing interests.

\section{Author details}

${ }^{1}$ Department of Radiation Oncology, First Affiliated Hospital of Guangxi Medical University, Nanning, Guangxi, China; ${ }^{2}$ Department of Radiation Oncology, Liuzhou people's Hospital, Liuzhou, Guangxi, China

\section{References}

1. Siegel RL, Miller KD, Jemal A. Cancer statistics, 2018. CA Cancer J Clin. 2018;68:7-30.

2. Peters WA 3rd, Liu PY, Barrett RJ 2. Concurrent chemotherapy and pelvic radiation therapy compared with pelvic radiation therapy alone as adjuvant therapy after radical surgery in high-risk early-stage cancer of the cervix. J Clin Oncol. 2000;18:1606-13. nd, et al.

3. Morris M, Eifel PJ, Lu J, et al. Pelvic radiation with concurrent chemotherapy compared with pelvic and para-aortic radiation for high-risk cervical cancer. N Engl J Med. 1999;340:1137-43.

4. Keys HM, Bundy BN, Stehman FB, et al. Cisplatin, radiation, and adjuvant hysterectomy compared with radiation and adjuvant hysterectomy for bulky stage IB cervical carcinoma. N Engl J Med. 1999;340:1154-61.

5. Rose PG, Bundy BN, Watkins EB, et al. Concurrent cisplatin-based radiotherapy and chemotherapy for locally advanced cervical cancer. N Engl J Med. 1999;340:1144-53.

6. Whitney CW, Sause W, Bundy BN, et al. A randomized comparison of fluorouracil plus cisplatin versus hydroxyurea as an adjunct to radiation therapy in stages IIB-IVA carcinoma of the cervix with negative para-aortic lymph nodes: A Gynecologic Oncology Group and Southwest Oncology Group Study. J Clin Oncol. 1999;17:1339-48.

7. Vale C, Tierney JF, Stewart LA, et al. Reducing uncertainties about the effects of chemoradiotherapy for cervical cancer: A systematic review and meta-analysis of individual patient data from 18 randomized trials. J Clin Oncol. 2008;26:5802-12.

8. Koh WJ, Greer BE, Abu-Rustum NR, et al. Cervical cancer, version 2.2015. J Natl Compr Canc Netw. 2015;13:395-404.

9. Katsumata $\mathrm{N}$, Yoshikawa $\mathrm{H}$, Kobayashi $\mathrm{H}$, et al. Phase III randomized controlled trial of neoadjuvant chemotherapy plus radical surgery vs radical surgery alone for stages IB2, IIA2, and IIB cervical cancer: a Japan Clinical Oncology Group trial (JCOG 0102). Br J Cancer. 2013;108:1957-63.

10. de Azevedo CRAS, Thuler LCS, de Mello MJG, et al. Phase II trial of neoadjuvant chemotherapy followed by chemoradiation in locally advanced cervical cancer. Gynecol Oncol. 2017;146:560-5.

11. Koensgen D, Sehouli J, Belau A, et al. Clinical Outcome of Neoadjuvant Radiochemotherapy in Locally Advanced Cervical Cancer: Results of an Open Prospective, Multicenter Phase 2 Study of the North-Eastern German Society of Gynecological Oncology. Int J Gynecol Cancer. 2017;27:500-6. 
12. Tanioka M, Yamaguchi S, Shimada M, et al. Cisplatin with dose-dense paclitaxel before and after radical hysterectomy for locally advanced cervical cancer: a prospective multicenter phase II trial with a dose finding study. Med Oncol. 2017;34:134-42.

13. Zhao H, He Y, Yang SL, et al. Neoadjuvant chemotherapy with radical surgery vs radical surgery alone for cervical cancer: a systematic review and meta-analysis. Onco Targets Ther. 2019;12:1881-91.

14. Gupta S, Maheshwari A, Parab P, et al. Neoadjuvant Chemotherapy Followed by Radical Surgery Versus Concomitant Chemotherapy and Radiotherapy in Patients With Stage IB2, IIA, or IIB Squamous Cervical Cancer: A Randomized Controlled Trial. J Clin Oncol. 2018;36:1548-55.

15. Yan W, Si L, Ding Y, et al. Neoadjuvant chemotherapy does not improve the prognosis and lymph node metastasis rate of locally advanced cervical squamous cell carcinoma: A retrospective cohort study in China. Medicine. 2019;98:e17234-8.

16. Zhao H, He Y, Zhu LR, et al. Effect of neoadjuvant chemotherapy followed by radical surgery for FIGO stage IB2/IIA2 cervical cancer: A multi-center retrospective clinical study. Medicine. 2019;98:e156048.

17. Chen $\mathrm{H}$, Liang $\mathrm{C}$, Zhang $\mathrm{L}$, et al. Clinical efficacy of modified preoperative neoadjuvant chemotherapy in the treatment of locally advanced (stage IB2 to IIB) cervical cancer: randomized study. Gynecol Oncol. 2008;110:308-15.

18. Yang Z, Chen D, Zhang J, et al. The efficacy and safety of neoadjuvant chemotherapy in the treatment of locally advanced cervical cancer: A randomized multicenter study. Gynecol Oncol. 2016;141:231-9.

19. Kim HS, Sardi JE, Katsumata N, et al. Efficacy of neoadjuvant chemotherapy in patients with FIGO stage IB1 to IIA cervical cancer: an international collaborative meta-analysis. Eur J Surg Oncol. 2013;39:115-24.

20. Marchetti C, Fagotti A, Tombolini V, et al. Survival and toxicity in neoadjuvant chemotherapy plus surgery versus definitive chemoradiotherapy for cervical cancer: A systematic review and metaanalysis. Cancer Treat Rev. 2020;83:101945-56.

21. Fyles $A$, Keane TJ, Barton M, et al. The effect of treatment duration in the local control of cervix cancer. Radiother Oncol. 1992;25:273-9.

22. Girinsky T, Rey A, Roche B, et al. Overall treatment time in advanced cervical carcinomas: a critical parameter in treatment outcome. Int J Radiat Oncol Biol Phys. 1993;27:1051-6.

23. Lanciano RM, Pajak TF, Martz K, et al. The influence of treatment time on outcome for squamous cell cancer of the uterine cervix treated with radiation: a patterns-of-care study. Int J Radiat Oncol Biol Phys. 1993;25:391-7.

24. Perez CA, Grigsby PW, Castro-Vita H, et al. Carcinoma of the uterine cervix. I. Impact of prolongation of overall treatment time and timing of brachytherapy on outcome of radiation therapy. Int J Radiat Oncol Biol Phy. 1995;32:1275-88.

25. Petereit DG, Sarkaria JN, Chappell R, et al. The adverse effect of treatment prolongation in cervical carcinoma. Int J Radiat Oncol Biol Phys. 1995;32:1301-7. 
Figures

Figure 1.Comparison of overall survival among three therapies

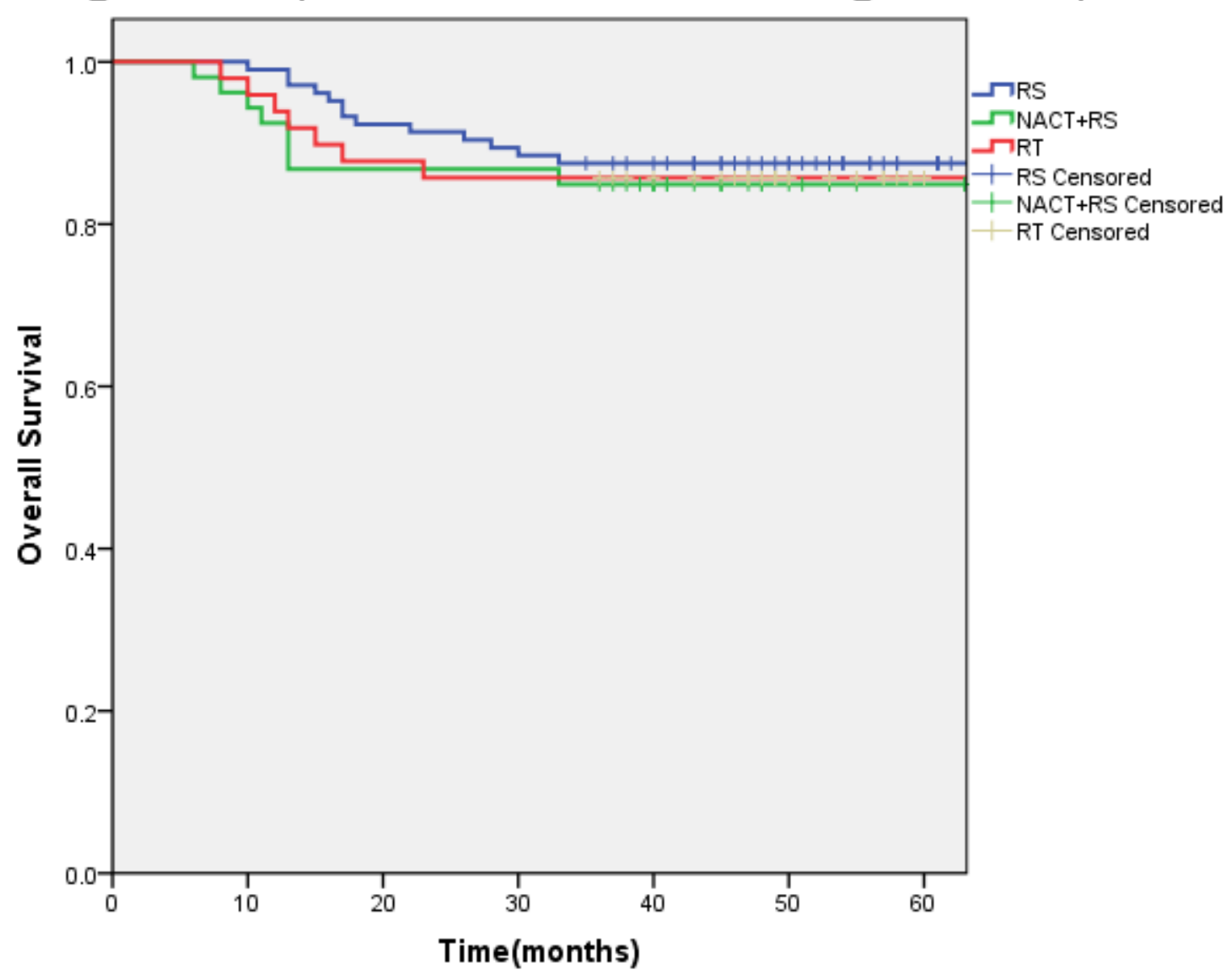

Figure 1

Overall survival comparison of the three groups (PST: $87.5 \%$, NACT+RS: $84.9 \%$, CCRT $85.7 \% ; P=0.843$ ). 
Figure 2.Comparison of disease free survival among three therapies

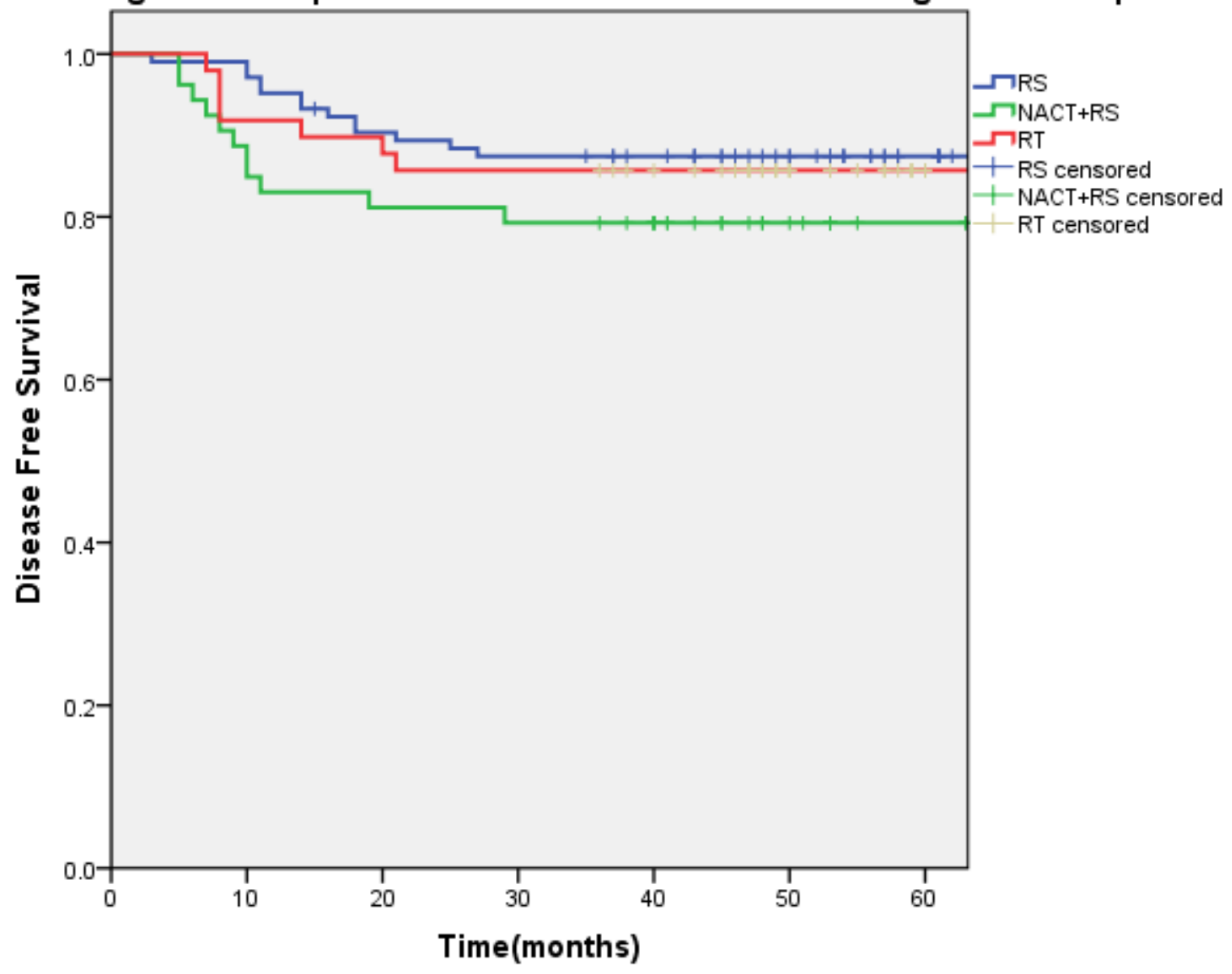

Figure 2

Disease-free survival comparison of the three groups (PST: $85.6 \%$, NACT+RS: $79.2 \%$, CCRT: $85.7 \%$; $\mathrm{P}=0.414)$. 